\title{
Numerical Simulation of Supersonic Aerodynamic Interaction of a Parachute System
}

\author{
By Xiaopeng Xue, Hiroto KoYAma* and Yoshiaki NAKAmURA \\ Department of Aerospace Engineering, Nagoya University, Nagoya, Japan
}

(Received May 22nd, 2012)

\begin{abstract}
In the present study the supersonic flow over rigid parachute models is studied by numerically solving the three-dimensional compressible Navier-Stokes equations at a free stream Mach number of 2. The parachute system employed here consists of a capsule and a canopy. Two models are considered: model A and model $\mathrm{B}$. The former is the same model as the experiment, where the canopy is connected with the capsule by a rod, and the whole system is supported by another rod, while the latter does not have these rods. The objective of the present study is to examine the flow field produced by these models, and analyze the effects of aerodynamic interaction such as shock/shock and wake/shock interactions on it. The numerical results show good agreement with the experimental data in the case of model A. In addition, it is found that the difference of flow features between models A and B is rather small. The unsteady flow pulsation phenomenon observed in this study can be demonstrated using three processes; the bow shock formed ahead of the capsule periodically inflates and laterally expands, which is caused by upstream propagation and lateral expansion of the complicated wake/rear shock and fore shock/rear shock interaction systems.
\end{abstract}

Key Words: Supersonic Parachute, Pulsation Mode, Wake/Shock Interaction, Shock/Shock Interaction

\section{Nomenclature}

$d \quad$ : diameter of capsule front surface

$d_{1} \quad$ : diameter of connecting rod

$d_{2} \quad$ : diameter of supporting rod

D : canopy diameter

$h \quad$ : canopy thickness

$M_{\infty} \quad$ : free stream Mach number

$V_{\infty} \quad$ : free stream velocity

$t \quad$ : non-dimensional time

$t_{1} \quad$ : dimensional time

$P \quad$ : dimensional pressure

$P_{\infty} \quad:$ free stream pressure

$P_{0} \quad:$ total pressure

$T_{0} \quad$ : total temperature

Re : Reynolds number

$T \quad$ : time period

$X \quad$ : axial distance from capsule front surface to canopy inlet

\section{Introduction}

In the Mars space mission, a supersonic parachute has been used to decelerate the spacecraft during entry, and for descent into the thin atmosphere based on its low mass and high ratio of aerodynamic drag. ${ }^{1)}$ However, in a full-scale experiment of the supersonic parachute system

(C) 2013 The Japan Society for Aeronautical and Space Sciences "Present address: Mitsubishi Heavy Industries Ltd., Ama District, Aichi, 490-1445, Japan in flight situations, because of the interaction of the capsule wake with the canopy bow shock, the drag decreases and flow instability increases. ${ }^{2)}$ Lingard et al. ${ }^{3)}$ have used the fluid structure interaction (FSI) to simulate a flexible supersonic parachute and examined the effects of Mach number and the trailing distance on the parachute. Barnhardt et al. ${ }^{4)}$ presented a numerical simulation of a rigid parachute model using the detached-eddy simulation method, and found that the time-dependent deficit in the wake interacts with the canopy bow shock, causing the flow field around the parachute to become highly unsteady.

However, while reducing the trailing distance, $X / d$, between the capsule and canopy in supersonic flow, another aerodynamic interaction occurs, where the shock wave ahead of the capsule interacts with the shock wave ahead of the canopy. Shock/Shock interaction in a two -body configuration often leads to critical aerodynamic and aerothermodynamic problems for spacecraft in supersonic flight conditions. ${ }^{5}$

In this study a rather small trailing distance is considered, so that the flow field around a parachute model shows complicated wake/shock and shock/shock interactions. In order to fully understand the flow field and investigate the effects of such wake/shock and shock/shock interaction on the flow instability, numerical simulation is carried out for three-dimensional rigid models at a free stream Mach number of 2.0. The computational results obtained for model A are validated by comparisons with experimental data measured at the ISAS/JAXA supersonic tunnel. 


\section{Method of Simulation}

\subsection{Model to be simulated}

The parachute system employed here consists of a capsule and a canopy. For comparisons with the experiment, a rigid body parachute canopy is considered, and the suspension lines are not taken into account. Two models are employed: models $\mathrm{A}$ and $\mathrm{B}$, which are shown in Fig. 1. In model $A$ the capsule and canopy are connected by a rod, all of which is supported at the top of the canopy by a thicker rod to the wind tunnel model support system; this configuration is the same as the model used in the experiment. On the other hand, model B is basically close to model A except for having no rods.

It should be noted that the capsule takes a conical form with a half-cone angle of $20 \mathrm{deg}$ and a front diameter of $0.20 D$ (see Fig.1).

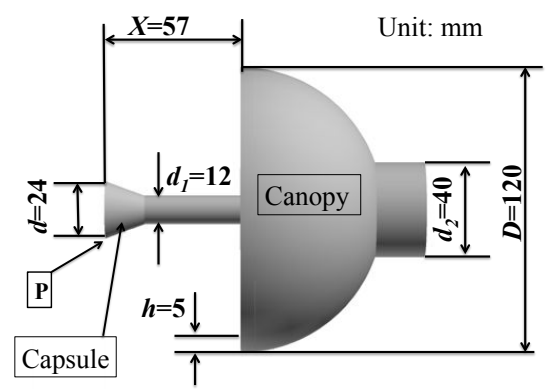

a) Model $\mathrm{A}$

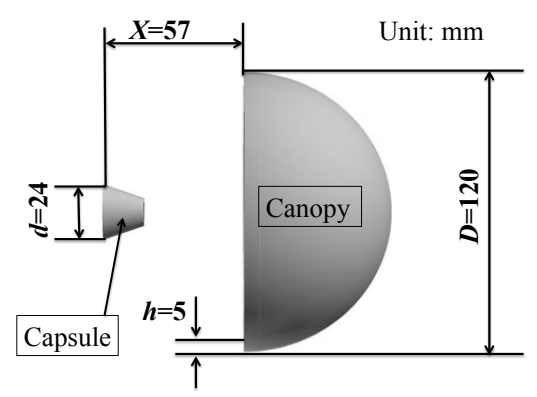

b) Model B

Fig. 1. Models used in the present computation period.

\subsection{Computational conditions}

The conditions for the free stream in computation are the same as those in the experiment, which are listed in Table 1.

Table 1. Flow conditions.

\begin{tabular}{ccccc}
\hline$M_{\infty}$ & $R e$ & $P_{0}$ & $P_{\infty}$ & $T_{0}$ \\
\hline 2.0 & $2.04 \times 10^{7} \mathrm{~m}^{-1}$ & $166 \mathrm{kPa}$ & $21.0 \mathrm{kPa}$ & $298 \mathrm{~K}$ \\
\hline
\end{tabular}

\subsection{Numerical method}

The calculations are performed using a parallel in-house structured single-block code, where the three-dimensional compressible Navier-Stokes equations are solved to simulate the supersonic flow field around parachute models. To evaluate the inviscid fluxes, the simple high-resolution upwind scheme (SHUS) ${ }^{6)}$ is adopted. The accuracy of this scheme is improved using the 3rd MUSCL scheme with the Van Albada flux limiter.
On the other hand, the viscous terms are calculated using the usual 2nd order central differencing scheme. The coefficient of viscosity is computed according to Sutherland's law. The numerical code is featured by the 3rd total variation diminishing Runge-Kutta scheme to obtain time accurate results in unsteady calculations.

We do not use any turbulence models in the present study, because till now most of the algebraic turbulence models have been quite unreliable for separation flows. ${ }^{7)}$ In addition, in terms of initial conditions, each variable takes its free stream value, while in terms of boundary conditions, non-slip and adiabatic conditions are imposed at body surfaces.

\subsection{Grid generation}

Owing to the axisymmetric configuration in the present problem, the single-block structured grid is created using a meridional plane. Figure 2 shows the 3D view of these grids.

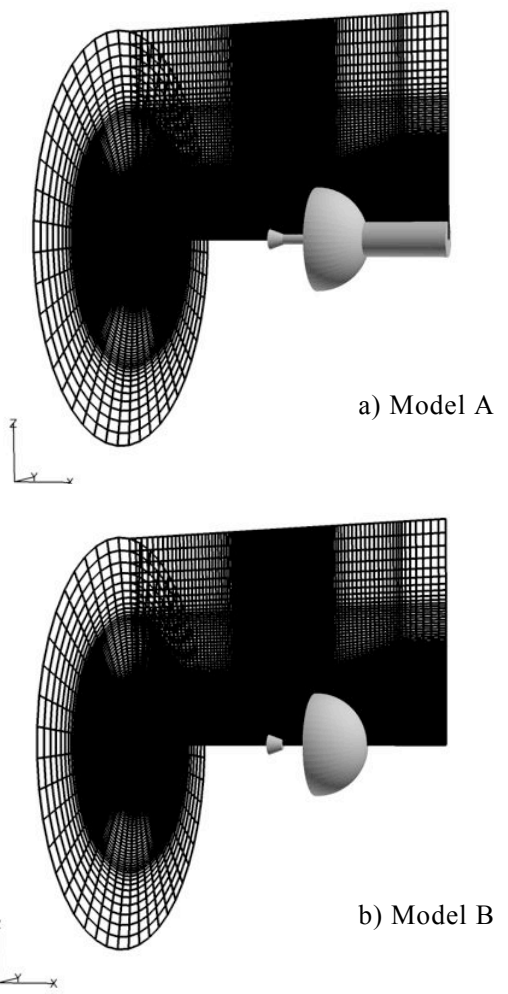

Fig. 2. Grids for parachute models.

\subsection{Grid convergence}

Three grids with a different number of points listed in Table 2 are employed to examine the grid convergence. The pressure histories for point $\mathrm{P}$ at the edge of capsule (see Fig.1) are shown in Fig. 3 at free stream Mach of 2.0. In this figure, the non-dimensional time is defined as $t=t_{1} * V_{\infty} / D^{8)}$. By and large, the pressure time histories are almost identical in terms of both pressure amplitude and time period. It is obvious that the resolution of the coarse grid is not enough to capture the appropriate unsteady flow. The pressure results of the medium and fine grids seem to agree reasonably, providing comparable values in terms of pressure amplitude and time period. Therefore, henceforth, results of the medium grid will be shown in this paper. 
X. XuE et al.: Numerical Simulation of Supersonic Aerodynamic Interaction of a Parachute System

Table 2. Grid used for grid convergence.

\begin{tabular}{c|c}
\hline Type of grid & Grid number \\
\hline Coarse & 542,640 \\
\hline Medium & $3,296,720$ \\
\hline Fine & $5,897,028$ \\
\hline
\end{tabular}

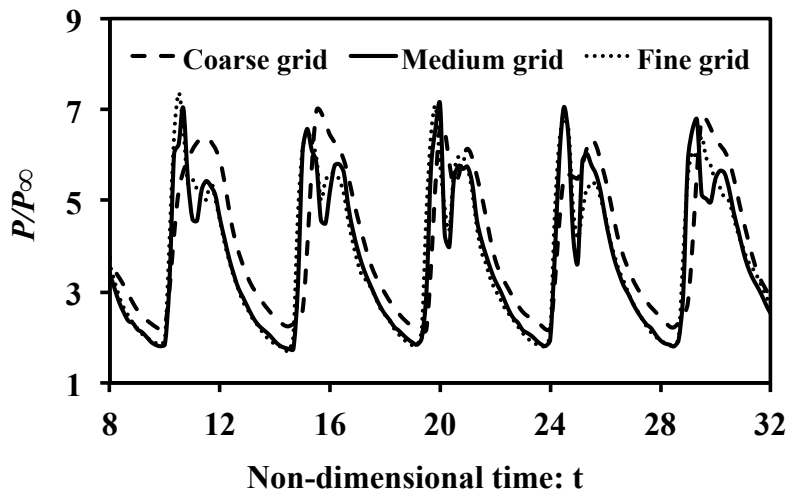

Fig. 3. Pressure change due to grid.

\subsection{Pressure sensor in the experiment}

The pressure sensor used in this experiment is PA-2KB made by Kyowa Electronic Instruments Co., Ltd. . It can precisely measure pressure fluctuations ranging from absolute zero (vacuum pressure) to $0.2 \mathrm{MPa}_{\mathrm{abs}} \cdot{ }^{9)}$ It should be noted that its measurement accuracy is $\left.\pm 0.2 \mathrm{kPa},{ }^{9}\right)$ and the main specifications of this pressure sensor are shown in Table 3.

Table 3. Specifications of the pressure sensor used in this study. ${ }^{9)}$

\begin{tabular}{|l|l|}
\hline Model & PA-2KB \\
\hline Rated capacity & $200 \mathrm{kPa}_{\text {abs }}\left(2.039 \mathrm{kgf} / \mathrm{cm}_{\text {abs }}^{2}\right)$ \\
\hline $\begin{array}{l}\text { Natural frequency } \\
\text { (approx.) }\end{array}$ & $5 \mathrm{kHz}$ \\
\hline Non-linearity & $\pm 0.1 \% \mathrm{RO}$ \\
\hline Hysteresis & $\pm 0.1 \% \mathrm{RO}$ \\
\hline Rated output & $2 \mathrm{mV} / \mathrm{V}\left(4,000 \times 10^{-6}\right.$ strain $)$ or higher \\
\hline
\end{tabular}

\subsection{Validation of the numerical method}

In both the experiment and Computational fluid dynamics (CFD), the shock wave envelopes exhibit cycle-to-cycle variations. As a result, the oscillation frequency of the flow can be described by Strouhal number, ${ }^{10)}$ which is adopted to make a quantitative comparison (see Table 4). The Strouhal number in the simulation is close to that of the experiment.

In addition, as the frequency of the pressure sensor used in the experiment is rather low (see Table 3), it could not capture unsteadiness of the pressure. Then, as shown in Fig. 5, a dotted line was drawn for a reference value based on the experimental data, which seems to correspond to the average value of unsteady pressure at point $\mathrm{A}$ on the inner surface of the canopy (see Fig. 4). It can be seen from this figure that the calculated pressure data (at point A) oscillates around this line.

The four representative instantaneous flow fields in the CFD in the case of model $\mathrm{A}$ are shown in Fig. 7 at free stream Mach number of 2.0 along with the corresponding shadowgraph pictures in the experiment. The four time locations of the CFD results are defined on the pressure trace of point $\mathrm{P}$ in Fig. 6. Comparing these figures, we can see that they are in reasonable agreement.

Table 4. Comparison of Strouhal numbers.

\begin{tabular}{c|c|c}
\hline Strouhal number & Experiment & CFD \\
\hline$\frac{D}{T \times V_{\infty}}$ & 0.2117 & 0.2139 \\
\hline
\end{tabular}

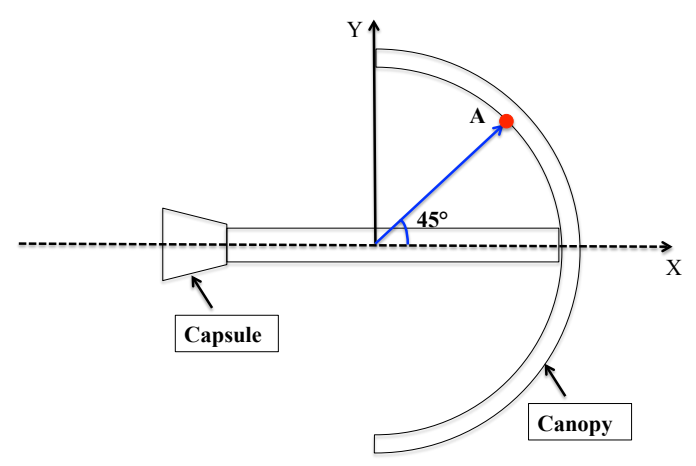

Fig. 4. Location of point A inside the canopy.

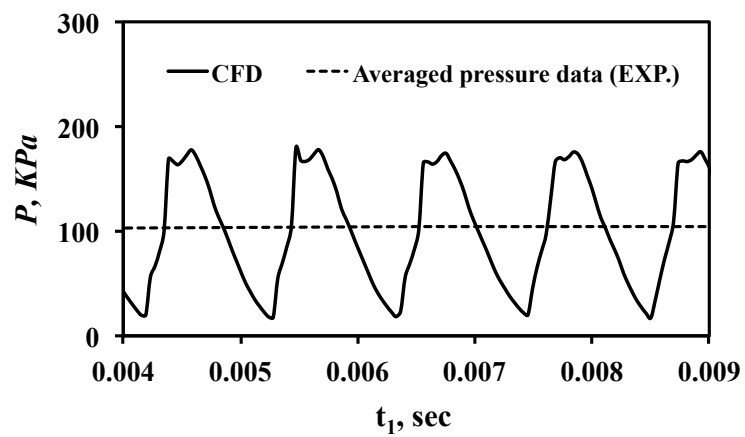

Fig. 5. Comparison of the experimental averaged pressure data and CFD time-resolved data of point A (see Fig. 4).

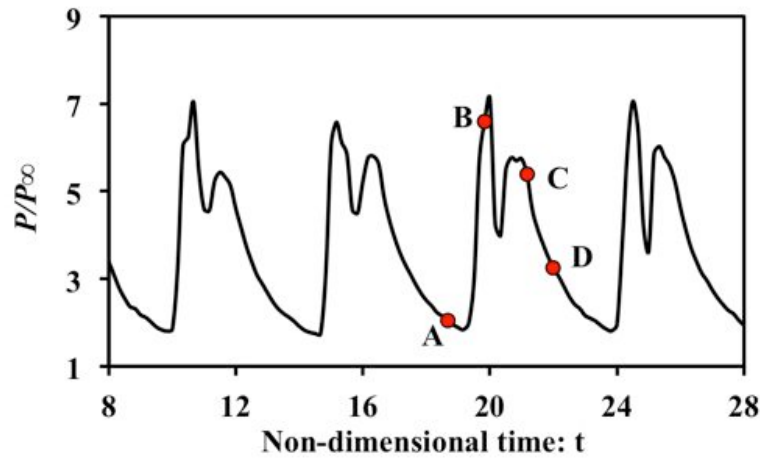

Fig. 6. Numerical pressure histories for point P (see Fig. 1).

Averaged pressure distributions on the inner surface of the canopy and the front surface of the capsule are plotted in Fig. 8, along with the experimental data, where $r$ represents the arc distance along the surface from the center, $L$ the maximum arc length of the canopy, and $d$ the diameter of the capsule frontal surface. It is seen from the figures that the computational results are in good 
quantitative agreement with the experimental data.
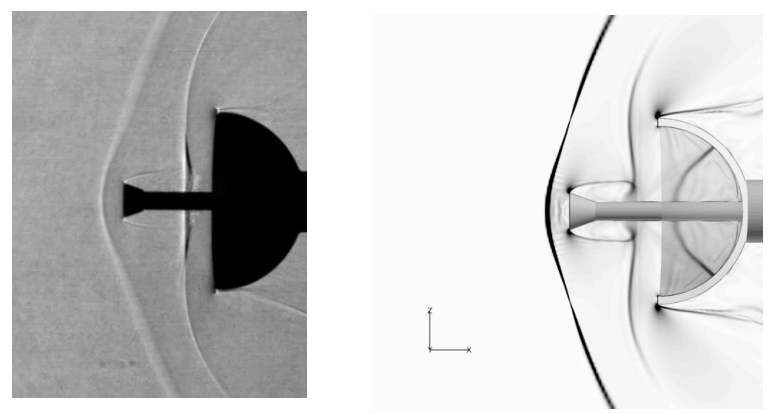

(A)
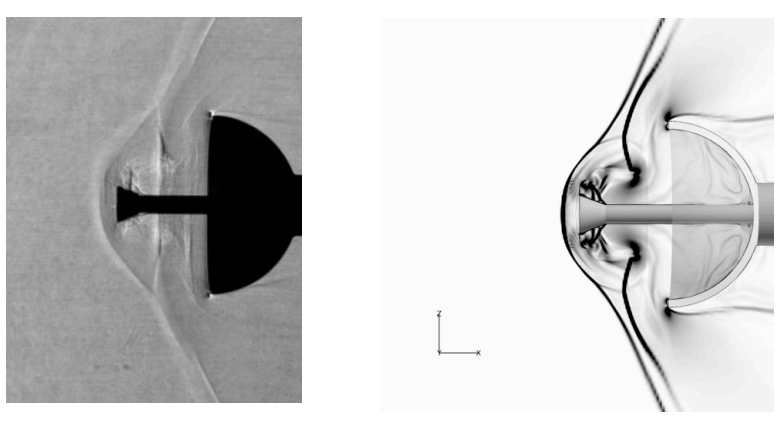

(B)

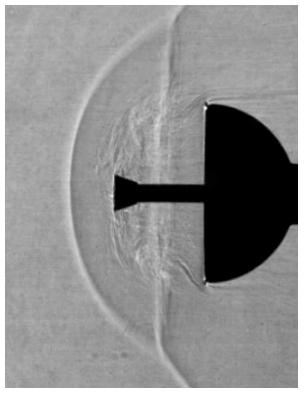

(C)
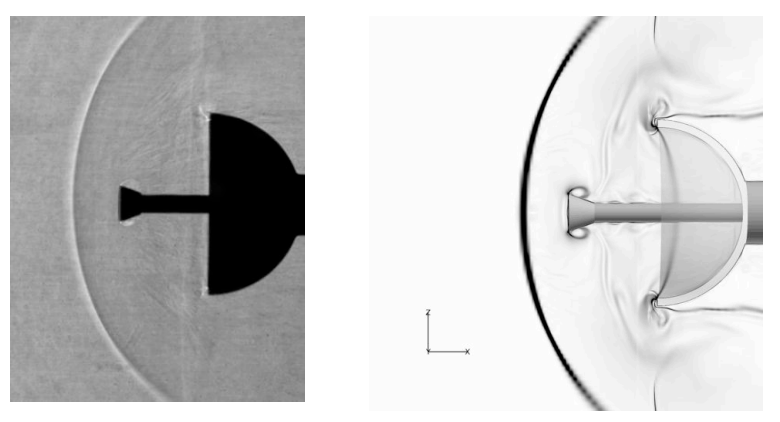

(D)

Fig. 7. Shadowgraphs pictures (left) and the corresponding density gradient contours in simulation (right).

\section{Results and Discussion}

\subsection{Unsteady aerodynamic interactions}

From Fig. 7, it is found that the bow shock (foreshock) formed ahead of the capsule periodically inflates and laterally expands, including a hemisphere shape. This unsteady flow mode is called "pulsation" mode. ${ }^{11)}$ In order to investigate the mechanism of this pulsation mode for the parachute system, due to the complicated flow field, the typical number of frames per pulsation cycle cannot be less than $10 .^{8,12)}$ Following the method of analyzing this flow mode proposed by Feszty et al., ${ }^{8)}$ in this study 14 frames per period were chosen from the CFD results to make two different types of flow visualization concurrently: Mach number contours and pressure contours. The former can demonstrate shear layer and boundary layer separation, while the latter can help to identify shock wave and vortex. ${ }^{8)}$ Here it should be noted that this paper focuses on analysis of the unsteady flow field produced by interactions between the capsule and canopy.

Figure 9 shows the 14 instantaneous flow field frames, where the time interval (dimensionless) between two neighboring frames is 0.3 . From the variation of the flow field during the pulsation cycle, it can be demonstrated using three processes; process 1 corresponds to frames 1-3, process 2 to frames 4-7, and process 3 to frames 8-14. Figure 10 shows the time variations of pressure at point $P$ and point $\mathrm{O}$ (see Fig. 11), which are used to define the time positions of the CFD results shown in Fig. 9, and properly identify the pressure difference between the canopy and the capsule from comparisons of these two pressure traces.

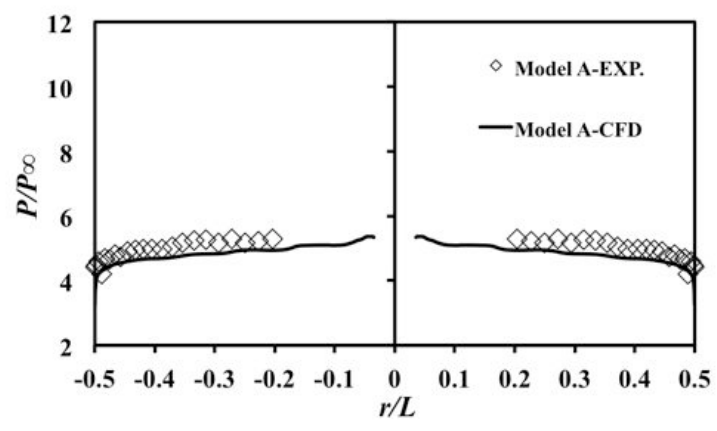

a) Inner surface of canopy

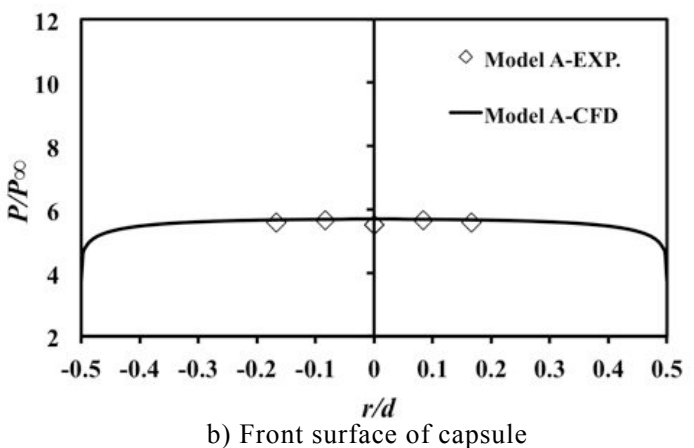

Fig. 8. Averaged pressure distribution on surfaces of model A.

As shown from Figs. 11 to 16, schematic symbols and schematic diagrams are used to show and explain the various flow characteristics appearing through the pulsation cycle, where $\mathrm{W}$ refers to the shock wave, $\mathrm{T}$ the triple point, $\mathrm{P}$ the separated region, $\mathrm{L}$ the shear layer, $\mathrm{V}$ the vortex region, and the number the order of its emergence. Since the geometry is axisymmetric and the flow fields are similar, those flow features are marked only once. In the following, the fore shock refers to the shock wave ahead of the capsule, and the rear shock refers 
X. XuE et al.: Numerical Simulation of Supersonic Aerodynamic Interaction of a Parachute System

to the shock wave ahead of the canopy.
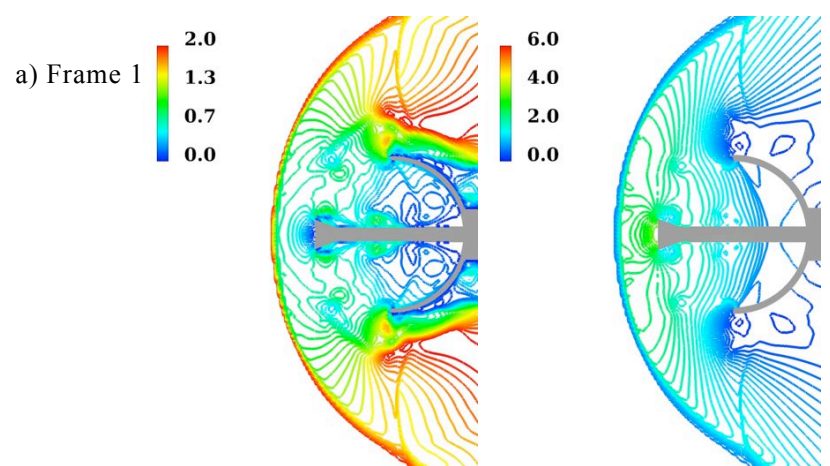

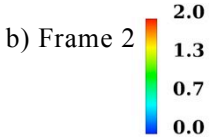

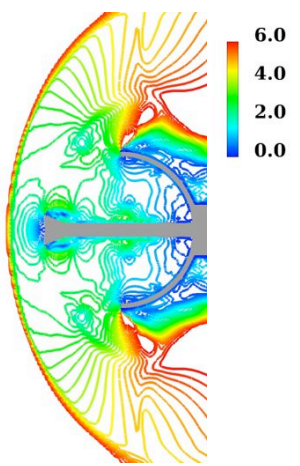

\begin{tabular}{l|l} 
c) Frame 3 & $\mathbf{2 . 0}$ \\
1.3 \\
0.7 \\
0.0
\end{tabular}

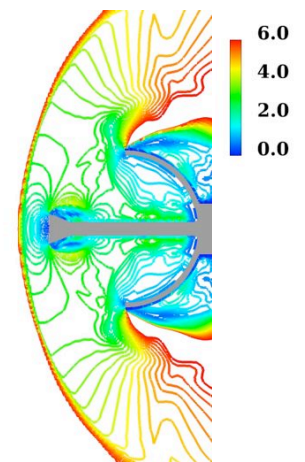

d) Frame $4\left[\begin{array}{l}2.0 \\ 1.3 \\ 0.7 \\ 0.0\end{array}\right.$

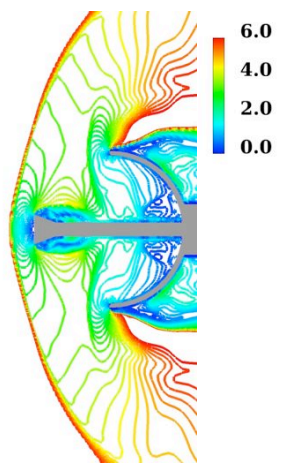

\begin{tabular}{l|l} 
e) Frame 5 & 2.0 \\
1.3 \\
0.7 \\
0.0
\end{tabular}

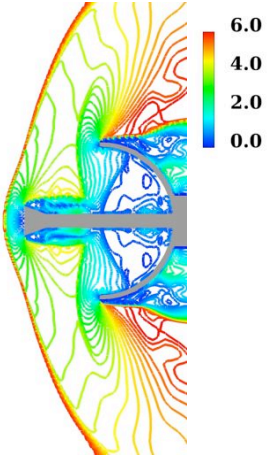

f) Frame $6 \mid \begin{aligned} & 2.0 \\ & 1.3 \\ & 0.7 \\ & 0.0\end{aligned}$
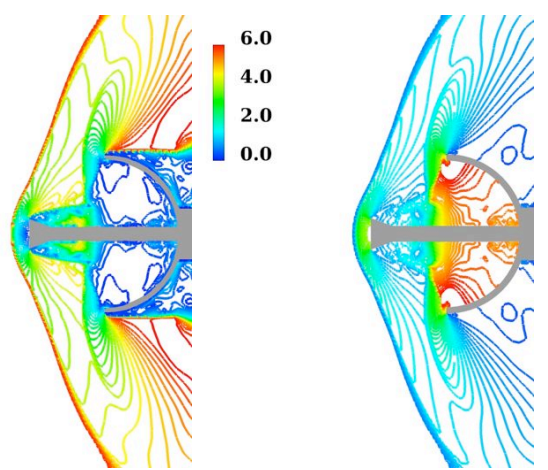

\begin{tabular}{l|l}
2.0 \\
g) Frame 7 \\
1.3 \\
0.7 \\
0.0
\end{tabular}
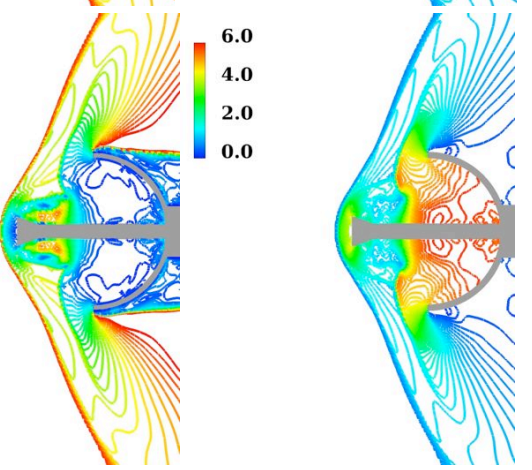

\begin{tabular}{l|l}
2.0 \\
h) Frame 8 & 1.3 \\
0.7 \\
0.0
\end{tabular}
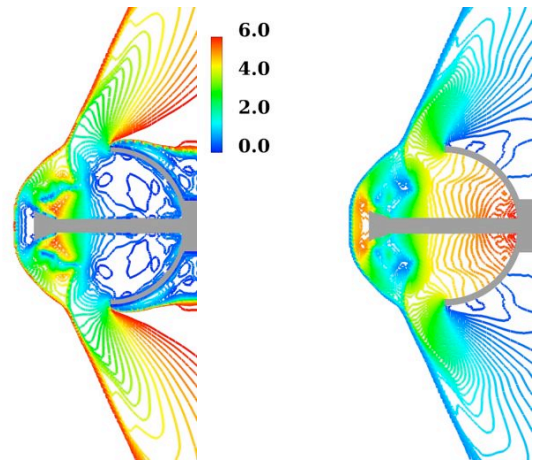

\begin{tabular}{l|l}
2.0 \\
1.3 \\
0.7 \\
0.0
\end{tabular}
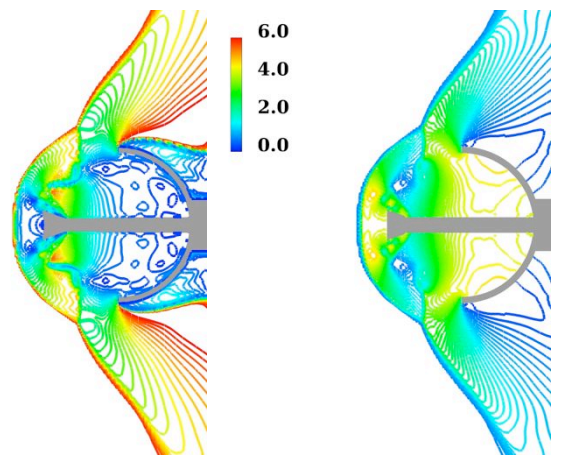

j) Frame 10 $\begin{aligned} & \mathbf{2 . 0} \\ & \mathbf{1 . 3} \\ & 0.7 \\ & 0.0\end{aligned}$
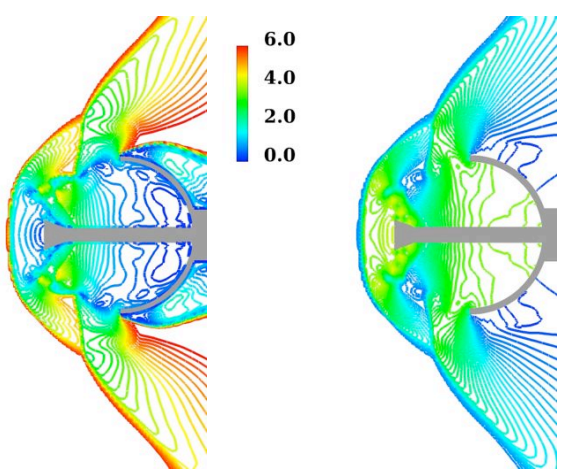

Fig. 9. Pulsation mode for Mach of 2.0: Mach number contours (left) and pressure contours (right). 


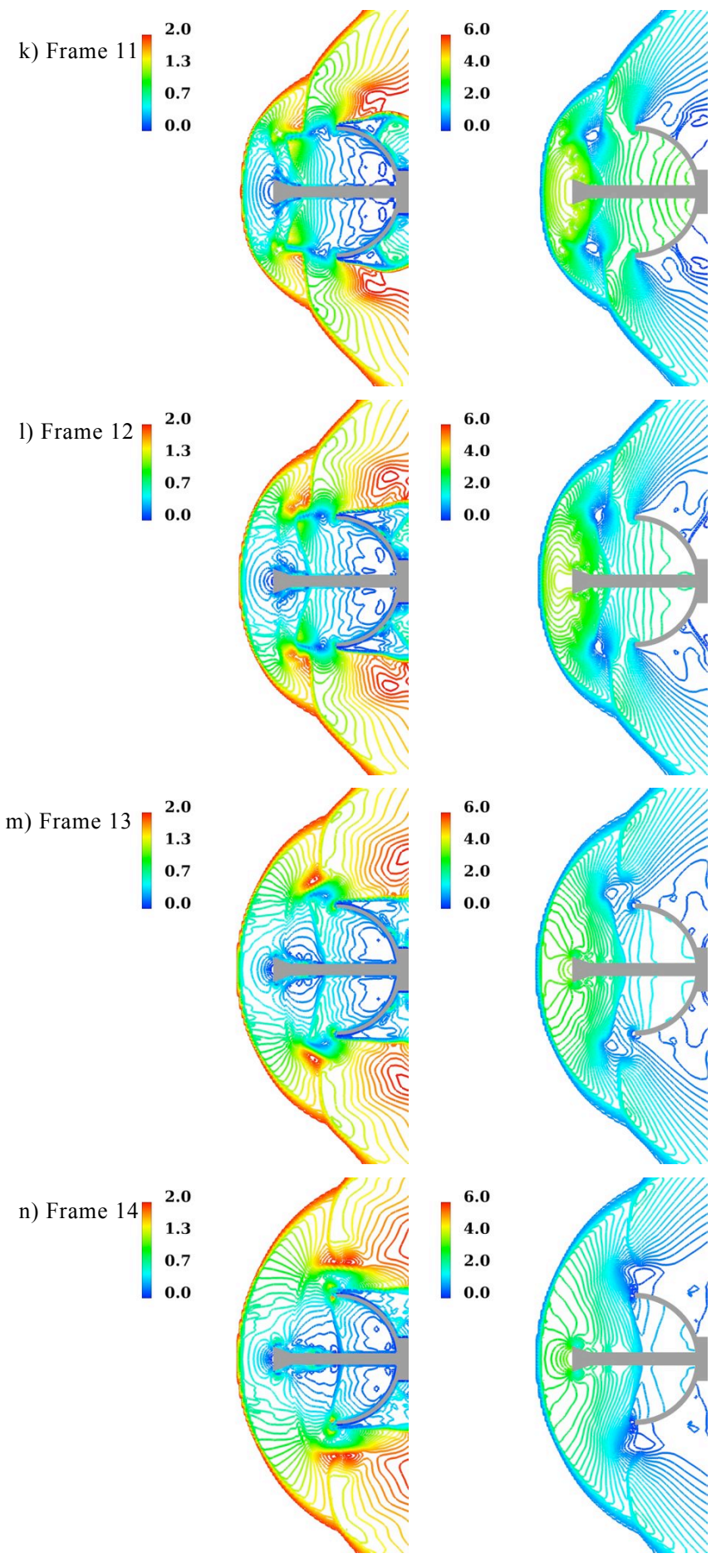

Fig. 9. Pulsation mode for Mach of 2.0: Mach number contours (left) and pressure contours (right). (Continued)

\section{A) Flow features of process 1}

This process corresponds to frames 1 to 3 in Fig. 9. The feature of this process is that no aerodynamic interactions occur during this process, where the bow shock, or the fore shock, is nearly stable, and the capsule wake and the canopy shock are formed.

In frame 1 of Fig. 9, bow shock W1 has expanded to its full extent and begins to go back to the capsule; the boundary layer separates from the edge of capsule, so that shear layer L1 and vortex V1 generate in the wake zone.
From Fig. 10, we can see that during frames 1-3, the pressure inside the canopy becomes almost minimum, while the pressure in front of the capsule is higher. This pressure difference makes shock W2 move toward the inside of canopy (see Figs. 9a and 9b). As it moves inward to the canopy, shock wave W2 interacts with the internal surface of the canopy, where the pressure starts to rise, leading to another shock W3 producing from there (see Figs. 9b and 11).

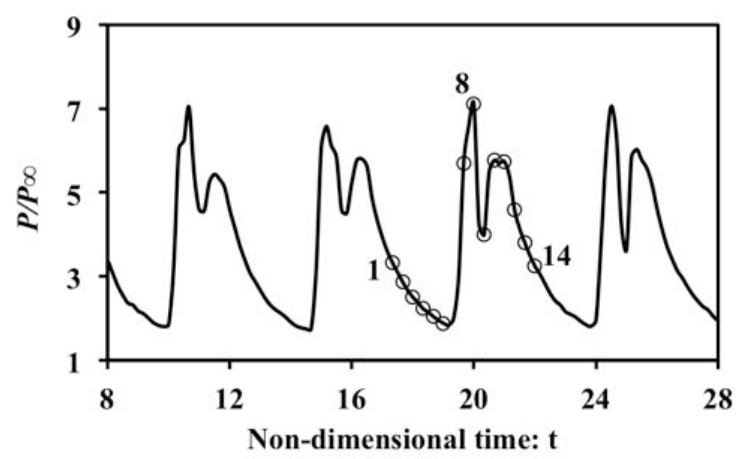

a) Point $\mathrm{P}$ at the edge of the capsule (see Fig. 11).

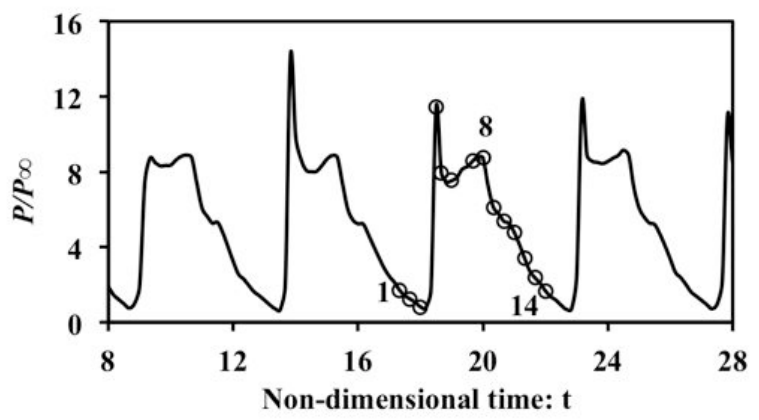

b) Point $\mathrm{O}$ at the corner of the canopy (see Fig.11).

Fig. 10. Time variations of pressure at two different places in Mach 2.0 pulsation case.

Moreover, as W2 goes downstream, it interacts with the boundary layer on the connecting rod and creates a separation region, P3, which gradually grows and moves forward. At the same time, a shear layer, L3, forms along the boundary of the separation zone P3 (see Figs. 9a, 9b and Fig. 11).

The flow characteristics of process 1 are that the gas inside the canopy is compressed by shock waves W2 and W3. When this compressed flow escapes from the edge of the canopy, it becomes supersonic (see Figs. 9b and 9c). As a result, the tip of canopy is exposed to this flow, so that a separation region, P2, forms. Following this, a shear layer, L2, forms in the wake region, leading to the development of a vortex region, V2 (see Fig. 11).

\section{B) Flow features of process 2}

This process corresponds to frames 4-7 in Fig. 9. The feature of this process is that aerodynamic interactions actually occur. Specifically the capsule wake interacts with the canopy shock, and the intersections go upstream.

It is seen from Fig. 10a that during frames 4-6, the pressure around the capsule becomes smaller and reaches a minimum at frame 6 . Consequently, shock wave W1 moves closer to the capsule. 
In frame 4 of Fig. 9, shock wave W2 almost reaches the corner, and then disappears. The pressure at the corner of the canopy becomes the first peak (see Fig. 10b), where shock wave W4 is produced (see Figs. 9d and 13). During this process, a definite vortex region, V3, can be observed at the corner of canopy, which forms behind shock wave W4 (see Fig. 13). This is produced by virtue of the impact of a penetrating flow with a recirculation flow. This suggests that the approaching expansion of the trapped air in the corner is generated not only by a mass influx from upstream, but also by the inflation due to its high pressure. The vortex V3 exits during almost the whole of process 2 .

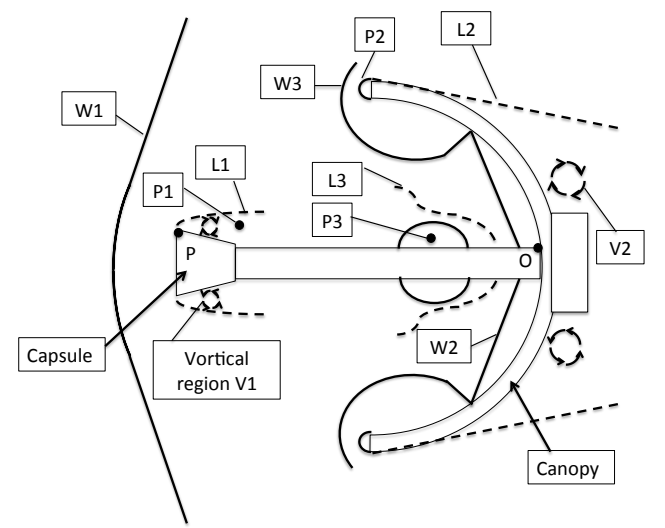

Fig. 11. Schematic of the flow field in process 1 , based on frame 2 of Fig. 9.

As shock wave W4 expands toward upstream, another vortex region, V4, is generated by the interaction of W4 with the inner surface of the canopy, which moves with W4 and reaches the edge of the canopy, as shown in frame 6 of Fig. 9. This leads to the creation of a vortical region, V6 (see Figs. 9f and 12).

In frame 5 of Fig. 9, shock wave W3 has already extended all over the entire canopy and has moved to the location of L1, interacting with it (see Fig. 9e).

In frame 6 of Fig. 9, shock wave W4 also reaches the interference region, and intersects $\mathrm{W} 3$ and $\mathrm{L} 1$, so that a weak oblique shock, W5, is generated (see Figs. 9f and 12). As a result of the wake/shock interaction, the flow velocity in the wake region increases to become supersonic, so that the vortex region, V1, disappears (see Fig. 9f).

It is seen from Fig.14 that the pressure behind shock wave W4 is higher than that in the wake region. Consequently, this pressure difference causes a strong pressure gradient, which induces a reverse flow; with the result that a definite vortex region, V5, develops at the foot of shock wave W4, as clearly observed in Fig. 14. This vortex region was also observed in other pulsation phenomenons. ${ }^{8,13)}$ The leading function of this flow phenomenon is a flow reversal. ${ }^{8)}$ The flow reverses and goes back to the wake region, through L1, W3 and W4 (see Fig. 14), which also causes vortex V3 to disappear, due to the lack of mass influx.

Frame 7 of Fig. 9 shows that shock wave W4 merges with $\mathrm{W} 3$, and that vortex region V4 disappears. As a result of high pressure within the canopy, both shock wave W3 and shear layer L1 are forced to move upstream and begin to expand laterally, so that the vortex region V5 grows. At the same time, shock wave W5 rapidly extends toward upstream, and merges with W1 (see Fig. 9h).

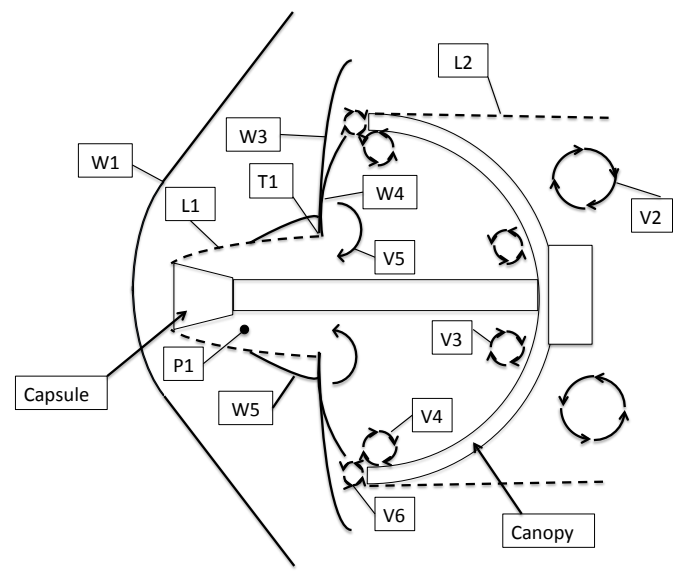

Fig. 12. Schematic of the flow field in process 2 , based on frame 6 of Fig. 9.

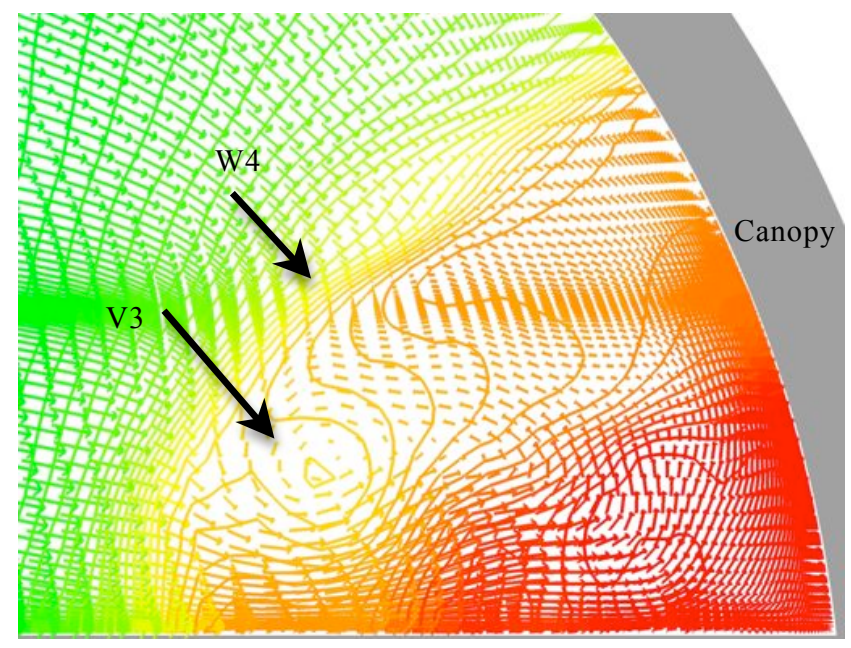

Fig. 13. Velocity vectors and pressure contours near the corner of the canopy in frame 4.

\section{C) Flow features of process 3}

Process 3 corresponds to frames 8-14 in Fig. 9. The feature of this process is that the triple shock systems inflate; i.e., the intersections of the fore and rear shocks extend more laterally, so that the fore shock takes a hemisphere shape.

In frames 8 and 9 of Fig. 9, shock wave W3 intersects $\mathrm{W} 1$, and generates a triple shock system consisting of $\mathrm{W} 1$, W3 and W6 (see Figs. 9h and 15), where the pressure inside the canopy reaches the second maximum (see Fig. $10 \mathrm{~b}$ ). It is seen from Fig. 9 that during process 3, the shock system of W1, W3 and W6 expand laterally all the time, where W1 takes a hemisphere shape. Due to this effect, the flow from separation region P1 (see Fig. 12) moves toward the bottom of the capsule, creating an oblique shock wave, W7 (see Figs. 9i and 15).

In frame 8 of Fig. 9 the pressure at the edge of capsule reaches a maximum (see Fig. 10a), leading to shear layer L1 becoming separated from the capsule (see Fig. 15). The movement of the shear layer can also be observed in 
another pulsation phenomenon ${ }^{8)}$ to respond to the expansion of the interference region. Here, as a consequence of this effect, the flow in separation region $\mathrm{P} 1$ reaches the front of the capsule, and collides with the flow originating from the capsule 's frontal area, causing a vortex region, $\mathrm{V} 7$, to be generated. In this process, the supersonic flow from P1 (see Fig. 12) has to be decelerated before entering the vortex region V7. Hence, the internal normal bow shock W8 is created between shear layer L1 and the capsule. As it interacts with the boundary layer on the neck of the capsule, another separation region, P4, occurs, and shear layer L4 forms at the boundary of P4 (see Figs. 9h and 15).

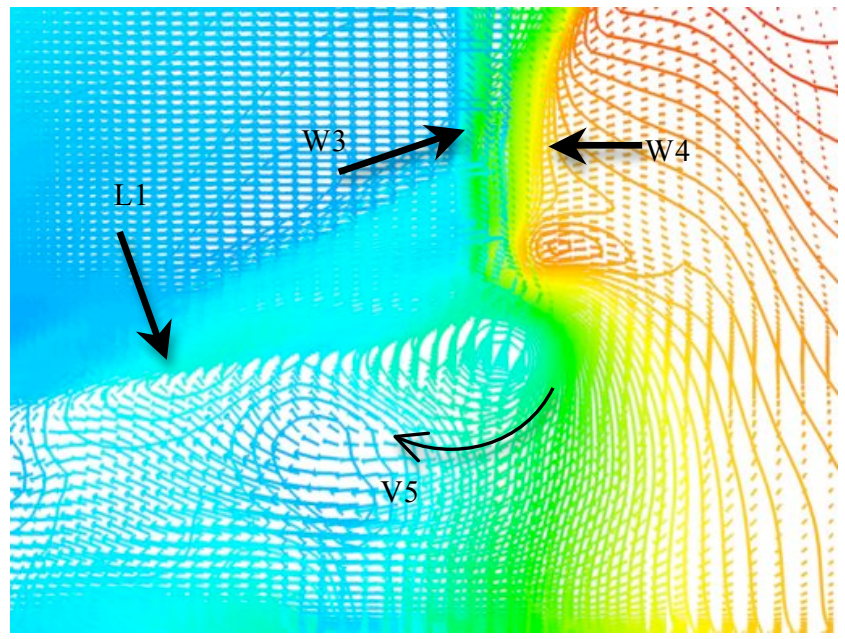

Fig. 14. Velocity vectors and pressure contours in the interaction region, based on frame 6 of Fig. 9.

In frame 9 of Fig. 9, shock wave W7 interacts with W8 and produces a new shock wave, W9, which constructs the second triple shock system (see Fig. 15).

In frames 10 and 11 of Fig. 9, as the pressure around the capsule remains high, the shock system with W7, W8 and W9 gradually strengthens and also expands laterally, which causes the enlargement of shear layer L4 and separation region $\mathrm{P} 4$ as well as the lateral movement of vortex region V7. In frame 12, the shock waves W8 and W9 merge with $\mathrm{W} 7$, and the vortex region V7 gradually disappears.

From frames 13 and 14 of Figs. 9 and 16, it is seen that the shock system W1-W3-W6 has reached the periphery of the canopy and continues to extend in the lateral direction. However, this system has already been weakened, and will disappear before going to the next frame 2 due to the low pressure within the canopy and around the capsule. As a result, shear layer L1 and vortex region V5 also vanish gradually. Moreover, we can see that shear layer L1 and vortex region V5 holds and develops during almost the whole interaction process. Thus, this is a key factor to drive the pulsation phenomenon considered here in this study. ${ }^{8)}$

As seen in frame 13 of Fig. 9, as shock wave W7 moves downstream due to the pressure difference, it interacts with the boundary layer on the connecting rod, so that a new separation region, $\mathrm{P} 6$, is created. At the same time the flow escapes from within the canopy, where the fluid is pressurized, and is accelerated in the vicinity of the canopy edge. Consequently, it collides with separated region P5 (see Fig. 15), and generates a vortex region, V8. In frame 14 of Fig. 9, shock wave W7 reaches to canopy edge, and vortex region V8 disappears.

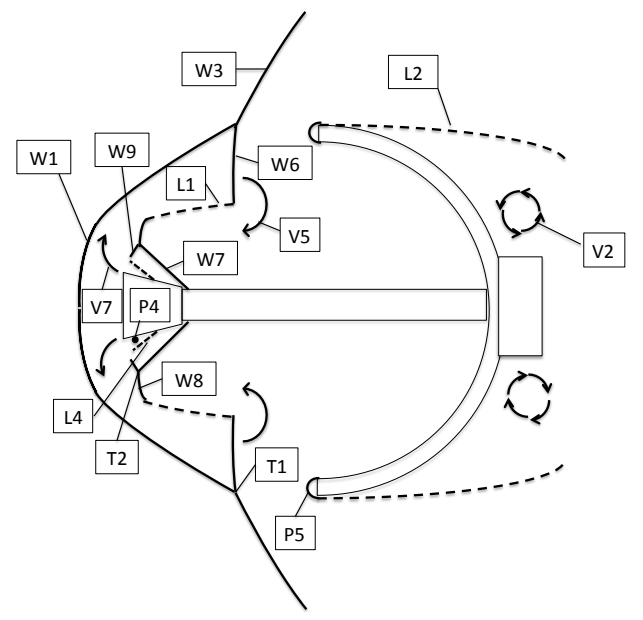

Fig. 15. Schematic of the flow field in the early stage of process 3, based on frames 8 and 9 of Fig. 9.

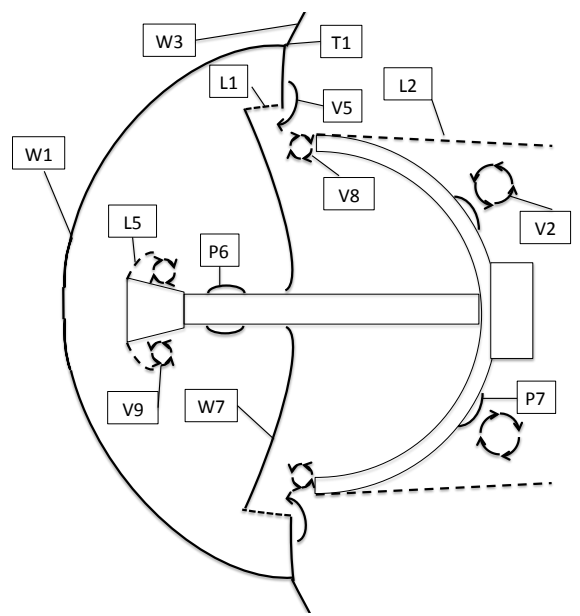

Fig. 16. Schematic of the flow field in the late stage of process 3, based on frame 13 of Fig. 9.

During process 3 , the unsteady flow upstream has a relatively large effect on the flow behind the canopy. In the early stage (see Fig. 15), the high pressure fluid escapes from inside the canopy, and moves downstream at supersonic speed, where shear layer L2 and vortex region V2 are depressed. On the other hand, in the late stage (see Fig. 16), as triple shock system T1 becomes weakened, the flow behind the canopy moves upstream, and collides with the outer surface of the canopy, causing a separation region, $\mathrm{P} 7$, to be generated.

\subsection{Effect of a connecting rod on the flow field}

Model A is used for comparisons with the experiment. In real conditions, the connecting rod is impossible. In order to examine the effect of the connecting rod on the aerodynamic interaction for the parachute, model $\mathrm{B}$ is employed. From Fig. 17, it is seen that the averaged 
X. XUE et al.: Numerical Simulation of Supersonic Aerodynamic Interaction of a Parachute System

pressure distribution on the typical surfaces of model B are in fair agreement with the ones of model A.

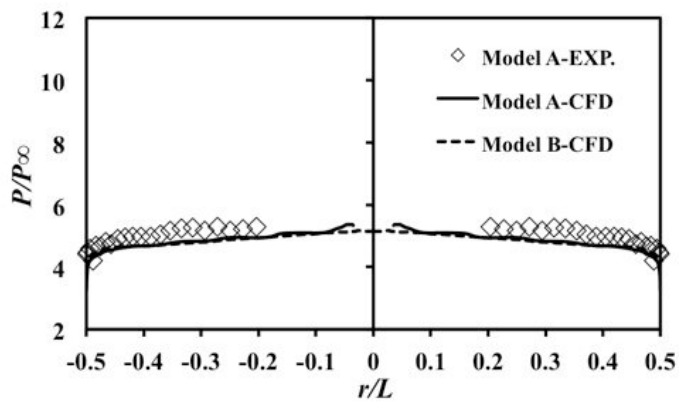

(a) Inner surface of canopy.

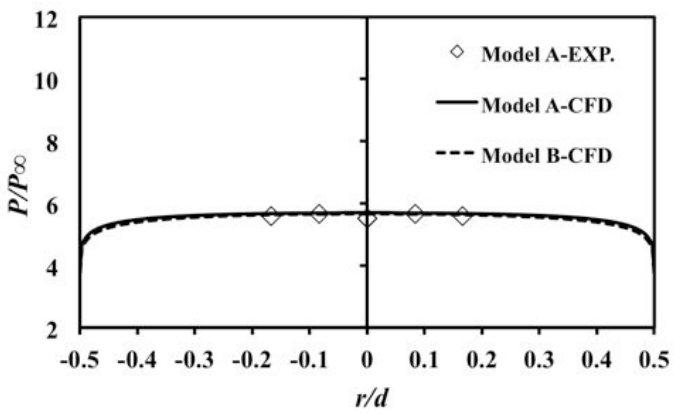

(b) Front surface of capsule.

Fig. 17. Comparison of averaged pressure distribution on typical surfaces between model A and model B.
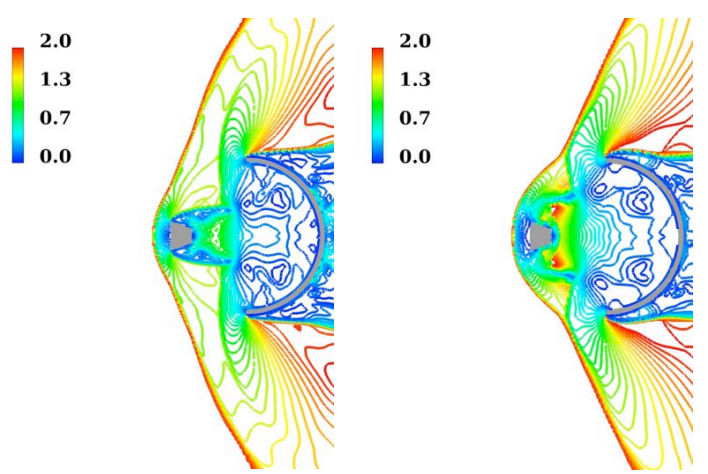

Fig. 18. Mach number contours of model B in two instantaneous flow fields: frame 6 (left) and frame 8 (right).

Two representative instantaneous aerodynamic interaction flow fields of model $\mathrm{B}$ are shown in Fig. 18. Compared with the same frames of model A, one can see that they are in good agreement. Except for some differences in the shock shape because of the connecting rod, the pulsation mechanism is identical to model A. Therefore, model A is reasonable and meaningful.

\subsection{Effect of Mach number}

In experiments, we can increase the Reynolds number by increasing the flow speed. However, it is difficult to change the Mach number while keeping the Reynolds number constant. On the contrary, in CFD, this can be easily achieved by changing the model size. ${ }^{14)}$ Here, based on this approach, computation is carried out for two cases: $M=1.6$ and $M=2.2$ under the same conditions as the case where $\mathrm{M}=2.0$.

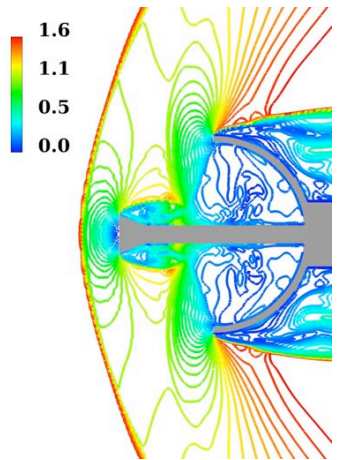

(A)

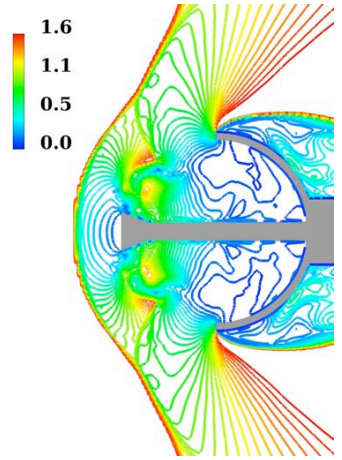

(B)
Fig. 19. Mach number contours of model A in two instantaneous flow fields for Mach of 1.6.

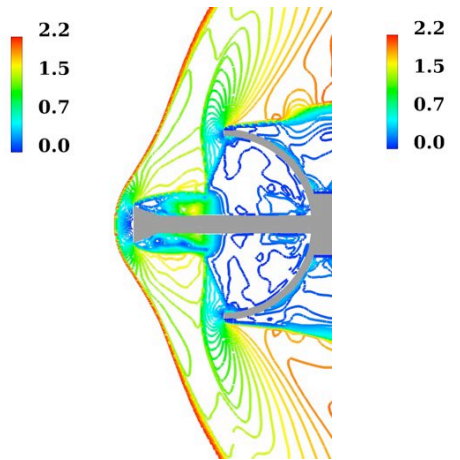

(A)

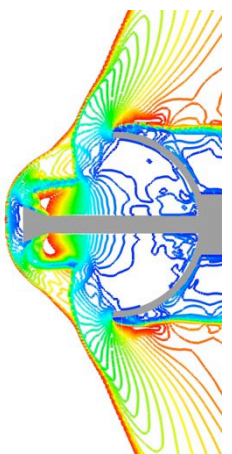

(B)
Fig. 20. Mach number contours of model A in two instantaneous flow fields for Mach of 2.2.

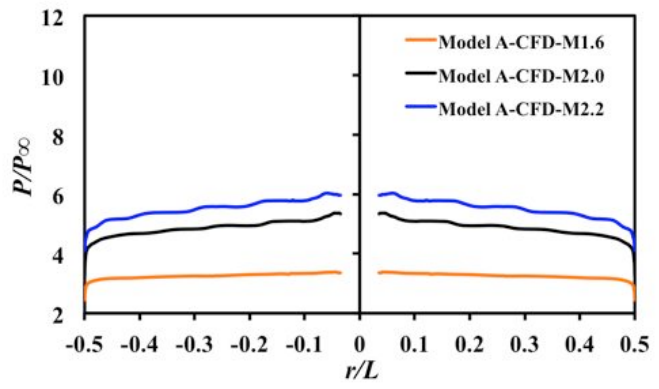

(A) Inner surface of canopy

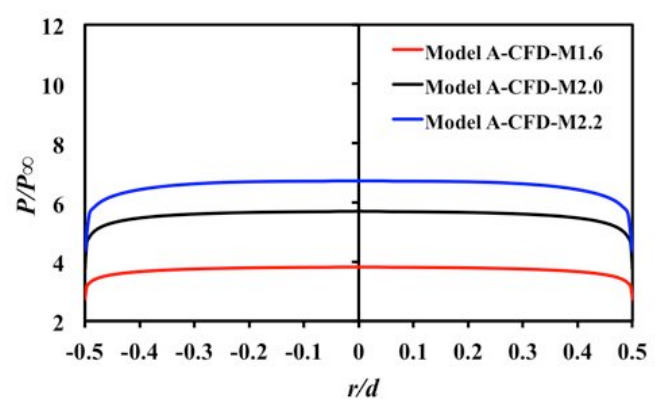

(B) Front surface of capsule

Fig. 21. Effect of Mach number on averaged pressure distribution on parachute surfaces.

From Figs. 19 and 20, it can be seen that for other Mach numbers the unsteady flow mode is consistent with that for Mach of 2.0. As the free stream Mach number is different, the period of a pulsation cycle becomes different. Here, two representative instantaneous flow fields with 
aerodynamic interactions similar to frames 6 and 8 for Mach of 2.0 are shown in Figs. 19 and 20. Comparing these three cases, it can be seen that when Mach number is 2.2, the bow shock ahead of the capsule moves closer toward the capsule, and becomes more conical in shape, leading to a decrease in the distance between the capsule bow shock and the canopy bow shock (see Fig. 20A). Consequently, coupling of the capsule wake with the canopy bow shock becomes stronger, and the region with aerodynamic interaction comes closer to the edge of the canopy (see Fig. 20B).

Figure 21 shows the averaged pressure distributions on the inner surface of canopy and the front surface of capsule. It can be seen that as the free stream Mach number increases, the pressure on the parachute surfaces become larger, which is caused by the stronger interference due to the higher energy flow.

\section{Conclusion}

The unsteady flow field with pulsation phenomenon, which occurs in the flow over a parachute model placed in a free stream with a Mach number of 2.0, was numerically simulated. The results obtained in this study can be summarized as follows:

- The computational results of model A, which has a connecting rod between the capsule and canopy, agreed with experimental data.

- When reducing the trailing distance between the capsule and canopy, complicated wake/rear shock and fore shock/rear shock interactions were observed.

- There were two key factors for the present pulsation phenomenon; one was the pressure difference between the capsule and the canopy, and the other the shear layer and vortex region produced by the wake/ rear shock and the fore shock/rear shock intersections.

- Judging from the results of models $A$ and $B$, the effects of the connecting rod on the flow field and pressure distribution on the body surfaces were rather small; the pulsation mechanism for model B was identical to that for model A.

- The unsteady flow pulsation phenomenon examined in this study can be demonstrated using three processes during one cycle. 1) At process 1, the shock ahead of the capsule was nearly stable. 2) At process 2, the capsule wake interacted with the canopy shock, and the movement of the wake-rear shock interaction was predominant. 3) At process 3, intersections of the fore and the rear shocks extended more laterally.

- In the unsteady flow pulsation mode, the bow shock formed ahead of the capsule periodically inflated and laterally expanded, which was caused by upstream propagation and lateral expansion of the complicated wake/rear shock and fore shock/rear shock interaction system.

- The mechanism for the unsteady flow mode was found to be consistent at supersonic speeds ranging from Mach of 1.6 to 2.2. As the free stream Mach number increased, the shock ahead of the capsule moved closer toward the capsule, and the capsule wake interacted more strongly with canopy bow shock, and the aerodynamic interaction region came closer to the edge of the canopy, which led to the pressure on the parachute surfaces becoming larger.

\section{Acknowledgments}

We are grateful to ISAS/JAXA for giving us permission to use the supersonic wind tunnel at ISAS/JAXA.

\section{References}

1) Karagiozis, K., Kamakoti, R. et al: A Computational Study of Supersonic Disk-Gap-Band Parachutes using Large-Eddy Simulation Coupled to a Structural Membrane, Journal of Fluids and Structures, 27(2011), pp.175-192.

2) Barnhardt,M.D.: Modeling and Simulation of High-Speed Wake Flows, Doctor thesis of the University of Minnesota, 2009, pp.102-106.

3) Lingard, J., Darley, M. et al.: Simulation of Mars Supersonic Parachute Performance and Dynamics, AIAA Paper 2007-2507, 2007.

4) Barnhardt, M., Drayna, T. et al.: Detached Eddy Simulations of the MSL Parachute at Supersonic Conditions, AIAA Paper 2007-2529, 2007.

5) Kitamura, K., Men'shov, I. and Nakamura, Y.: Shock/shock and Shock/Boundary-Layer Interactions in Two-Body Configurations, AIAA Paper 2005-4893, 2005.

6) Shima, E. and Jounouchi, T.: Roe of CFD in Aeronautical Engineering (No.14) -AUSM Type Upwind Schemes-, NAL-SP30, Proceedings of 13th NAL symposium on Aircraft Computational Aerodynamics, 1996, pp. 41-46.

7) Rahman, M. and Brebbia, C.A.: Advanced In Fluid Mechanics VIII, WIT Press, 2010, pp. 55-66.

8) Feszty, D., Badcock, K.J. and Richards, B.E.: Driving Mechanisms of High-Speed Unsteady Spiked Body Flows, Part 1: Pulsation Mode, AIAA J., 42(2004), pp. 95-106.

9) Soltec: Kyowa Pressure Transducers, CAT.204C-U3, p.16.

10) White, F.M.: Fluid Mechanics (4th ed.), McGraw Hill, 1999, pp. 295-297.

11) Panaras, A.G., Drikakis, D.: High-speed Unsteady Flows Around Spiked-Blunt Bodies, Journal of Fluid Mechanics, 632(2009), pp.69-96.

12) Kenworthy, M.: A Study of Unstable Axisymmetric Separation in High Speed Flows, Ph.D. Dissertation, Dept. of Aerospace and Ocean Engineering, Virginia Polytechnic Inst. and State Univ., Blacksburg, VA, 1978.

13) Zapryagaev, V.I. and Kavun, I.N.: Experimental Study of the Reverse Flow in the Forward Separation Region in a Pulsation Flow Around a Spiked Body, Journal of Applied Mechanics and Technical Physics, 48(2007), pp. 492-500.

14) Nonomura, T., Muranaka, H. and Fujii, K.: Computational Analysis of Mach Number Effects on the Edgetone Phenomenon, AIAA J., 48(2010), pp.1248-1251. 